\title{
Multi-point Taylor approximations in one-dimensional linear boundary value problems
}

\author{
José L. López ${ }^{1}$, Ester Pérez Sinusía ${ }^{1}$ and Nico M. Temme² \\ 1 Departamento de Ingeniería Matemática e Informática, \\ Universidad Pública de Navarra, 31006-Pamplona, Spain. ${ }^{2}$ CWI, P.O. Box 94079, 1090 GB Amsterdam, The Netherlands. \\ e-mail: jl.lopez@unavarra.es, ester.perez@unavarra.es, nicot@cwi.nl.
}

ABSTRACT. We consider second order linear differential equations in a real interval $I$ with mixed Dirichlet and Neumann boundary data. We consider a representation of its solution by a multi-point Taylor expansion. The number and location of the base points of that expansion are conveniently chosen to guarantee that the expansion is uniformly convergent $\forall x \in I$. We propose several algorithms to approximate the multipoint Taylor polynomials of the solution based on the power series method for initial value problems.

2000 AMS Mathematics Subject Classification: 34A25, 34B05, 41A58.

Keywords \& Phrases: second order linear differential equations, boundary value problem, Frobenius method, multi-point Taylor expansions.

\section{Introduction}

We consider boundary value problems of the form

$$
\left\{\begin{array}{l}
\varphi(x) y^{\prime \prime}+f(x) y^{\prime}+g(x) y=h(x) \quad \text { in }(0,1), \\
M Y=N
\end{array}\right.
$$

where

$$
M=\left(\begin{array}{llll}
M_{11} & M_{12} & M_{13} & M_{14} \\
M_{21} & M_{22} & M_{23} & M_{24}
\end{array}\right), \quad N=\left(\begin{array}{c}
N_{1} \\
N_{2}
\end{array}\right), \quad Y^{T}=\left(y(0), y^{\prime}(0), y(1), y^{\prime}(1)\right)
$$

$M_{i j}$ and $N_{i}$ are real numbers and $\operatorname{rank}(M)=2$. In the remaining of the paper we suppose that (1) has a unique solution.

Assume that $\varphi, f, g$ and $h$ are analytic at a point $x=c \in \mathbb{C}$ and denote by $D_{r}(c)$ the common disk of convergence of the Taylor series of those functions at $x=c$. If the radius of convergence $r$ is large enough such that $[0,1] \subset D_{r}(c)$ and $\varphi(x) \neq 0$ in that disk, then we can compute the Taylor expansion $y_{n}(x)$ of the solution at $x=c[2],[7]$ :

$$
y(x) \simeq y_{n}(x):=\sum_{k=0}^{n} a_{k}(x-c)^{k} .
$$


We equate to zero the Taylor coefficients at $x=c$ of $R(x):=\varphi(x) y_{n}^{\prime \prime}(x)+f(x) y_{n}^{\prime}(x)+g(x) y_{n}(x)-h(x)$ up to the order $n-2$. We obtain in this way a system of $n-1$ linear equations for the $n+1$ unknowns $a_{0}, a_{1}, a_{2}, \ldots, a_{n}$. This system is complemented with the two linear equations $M Y=N$. We obtain then a linear system of $n+1$ equations and $n+1$ unknowns $a_{0}, a_{1}, a_{2}, \ldots, a_{n}$, the solution of which gives an approximation to the Taylor polynomial $y_{n}(x)$, and then an approximation of the solution $y(x)$ of $(1)$ [2], [7]. When $M_{11}=M_{22}=1$ and the remaining $M_{i j}=0$ ((1) is an initial value problem), this is the well-known method of Frobenius [[6], chap. 0.2.2] the convergence of which has been demonstrated. On the other hand, as far as we know, the convergence of the method for the general boundary value problem (1) has not been proved.

When $[0,1]$ is not included in the disk $D_{r}(c)$, we can take several points $c_{k}$ (typically along the interval $[0,1])$ in such a way that $[0,1] \subset \bigcup_{k} D_{r_{k}}\left(c_{k}\right)$. Then, we essay a Taylor expansion of the solution at every such point $x=c_{k}$ and match these expansions at intersecting disks $D_{r_{k}}\left(c_{k}\right)$ [[5], Sec. 7]. In this way, we obtain an approximation of the solution of (1) in the form of a piecewise polynomial in several subintervals of $[0,1]$. Although this method gives an analytic approximation to the solution, this approximation is not uniform in the whole interval $[0,1]$ and the successive matching of expansions translates into numerical errors.

In the next section, we revisit the method considered in [2] and [7], proposing different algorithms and showing their convergence. In Section 3 we propose a different method by considering two-point Taylor expansions instead of the classical Taylor expansion. As a difference with the technique indicated in [5], this method gives a polynomial approximation uniform in $[0,1]$. The approximation by an $n$-points Taylor expansion is a straightforward generalization and we just give some indications in Section 4. A few remarks and comments are given in Section 5.

\section{A Taylor expansion of the solution at an arbitrary point}

Suppose that the functions $\varphi, f, g$ and $h$ are analytic in a disk $D_{r}(c)$ such that $[0,1] \subset D_{r}(c)$ and $\varphi$ not vanishing there (the real or complex point $c$ may or may not belong to the interval $[0,1]$ ). Then, the functions $\varphi, f, g$ and $h$, as well as $y$, have a Taylor expansion at $x=c$, which is convergent for $x \in[0,1]$. We propose the following algorithm to approximate $y$ :

Algorithm 1. Consider the auxiliary initial value problem:

$$
\left\{\begin{array}{l}
\varphi(x) y^{\prime \prime}+f(x) y^{\prime}+g(x) y=h(x) \quad \text { in }(0,1), \\
y(c)=a_{0}, \quad y^{\prime}(c)=a_{1}
\end{array}\right.
$$

where $a_{0}$ and $a_{1}$ are, at this moment, unknown parameters. In order to determine the solution $y$ of (2) we substitute its Taylor expansion at $x=c$ :

$$
y(x)=\sum_{k=0}^{\infty} a_{k}(x-c)^{k}
$$

and apply the standard method of Frobenius to the initial value problem (2). We equate to zero the Taylor coefficients at $x=c$ of the function $R(x):=\varphi(x) y^{\prime \prime}+f(x) y^{\prime}+g(x) y-h(x)$. We obtain in this way a recursion for $a_{k}$ of the form [[8], Sec. 4.2.4]

$$
a_{k}=\sum_{j=0}^{k-1} \alpha_{k, j} a_{j}+\beta_{k}, \quad k=2,3,4, \ldots
$$


where $\alpha_{k, j}$ and $\beta_{k}$ depend on the coefficients of the Taylor expansions of $\varphi, f, g$ and $h$ at $x=c$. From this recurrence relation we obtain the Taylor coefficients $a_{k}, k=2,3,4, \ldots$ of $y$ at $x=c$ as an affine combination of the two first coefficients $a_{0}$ and $a_{1}$ :

$$
a_{k}=A_{k} a_{0}+B_{k} a_{1}+C_{k}, \quad k=2,3,4, \ldots
$$

where $A_{k}, B_{k}$ and $C_{k}$ are functions of $\alpha_{k, j}$ and $\beta_{k}$.

We truncate the Taylor series of $y$ in (3) at a given $n \in \mathbb{N}$ to obtain

$$
y_{n}\left(x ; a_{0}, a_{1}\right):=\sum_{k=0}^{n} a_{k}(x-c)^{k},
$$

the Taylor polynomial of the solution $y$ of (2) with coefficients $a_{k}$ that are affine functions of $a_{0}$ and $a_{1}$. Then, we impose the boundary conditions over $y_{n}\left(x ; a_{0}, a_{1}\right)$ :

$$
M Y_{n}=N, \quad Y_{n}^{T}=\left(y_{n}\left(0 ; a_{0}, a_{1}\right), y_{n}^{\prime}\left(0 ; a_{0}, a_{1}\right), y_{n}\left(1 ; a_{0}, a_{1}\right), y_{n}^{\prime}\left(1 ; a_{0}, a_{1}\right)\right) .
$$

This is a linear system of two equations with two unknowns, $a_{0}$ and $a_{1}$, whose solution (when it exists and is unique) gives an approximation to $a_{0}$ and $a_{1}$ that we denote by $\tilde{a}_{0}$ and $\tilde{a}_{1}$ respectively. From these approximations $\tilde{a}_{0}$ and $\tilde{a}_{1}$ and (4) we obtain an approximation of any $a_{k}, k=2,3, \ldots, n$ that we denote by $\tilde{a}_{k}$. Then, we obtain the approximate Taylor polynomial of $y$ at $x=c$ and degree $n$ :

$$
\tilde{y}_{n}(x):=y_{n}\left(x ; \tilde{a}_{0}, \tilde{a}_{1}\right)=\sum_{k=0}^{n} \tilde{a}_{k}(x-c)^{k}
$$

as an approximation of the solution $y$ of (1).

Example 1. Consider the boundary value problem

$$
\left\{\begin{array}{l}
y^{\prime \prime}-(b-a)^{2}[a+(b-a) x] y=0 \quad \text { in }(0,1) \\
y(0)=\operatorname{Ai}(a), \quad y(1)=\operatorname{Ai}(b)
\end{array}\right.
$$

with $a<b$. We have $M_{11}=M_{23}=1$ and the remaining $M_{i j}=0 ; N_{1}=\operatorname{Ai}(a), N_{2}=\operatorname{Ai}(b), \varphi(x)=1$, $f=0, g(x)=-(b-a)^{2}[a+(b-a) x]$ and $h=0$. We consider the base point $c=1 / 2$. The unique solution of this problem is given in terms of the Airy function: $y(x)=\operatorname{Ai}(a+(b-a) x)$.

For several $n \in \mathbb{N}$, we seek for an approximation $\tilde{y}_{n}(x)$ of the Taylor polynomial $y_{n}(x)$ of $y(x)$ in the form explained above. Figure 1 illustrates the approximation $y(x) \simeq \tilde{y}_{n}(x)$ for some values of $n, a$ and $b$.

The proof of the convergence of Algorithm 1 is as follows. From the method of Frobenius, and taking into account equation (5) we know that, for given $\left(a_{0}, a_{1}\right)$, problem (2) has a unique solution in $D_{r}(c)$ of the form

$$
y\left(x ; a_{0}, a_{1}\right)=a_{0} u(x)+a_{1} v(x)+w(x) \quad \forall x \in[0,1],
$$

with

$$
u(x):=\sum_{k=0}^{\infty} A_{k}(x-c)^{k}, \quad v(x):=\sum_{k=0}^{\infty} B_{k}(x-c)^{k}, \quad w(x):=\sum_{k=0}^{\infty} C_{k}(x-c)^{k}
$$

and $A_{0}=B_{1}=1, A_{1}=B_{0}=C_{0}=C_{1}=0$. Observe that $w(x)$ is the unique solution of (2) for $a_{0}=a_{1}=0(w(x)=0$ if $h(x)=0)$ and $u(x)$ and $v(x)$ are two independent solutions of the differential equation in (2) with $h$ replaced by 0 . 


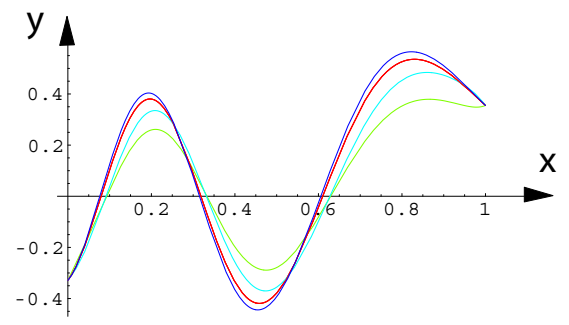

Figure 1. Plot of the exact solution of (7), $y(x)=A i(a+(b-a) x)$ (red), and the approximations $\tilde{y}_{16}(x)$ (green), $\tilde{y}_{18}(x)$ (blue) and $\tilde{y}_{20}(x)$ (dark blue) with $c=1 / 2, a=-6$ and $b=0$.

When we truncate these series at a given $k=n$, we obtain the Taylor approximation of degree $n$ at $x=c$ of $y\left(x ; a_{0}, a_{1}\right)$ :

$$
y\left(x ; a_{0}, a_{1}\right) \simeq y_{n}\left(x ; a_{0}, a_{1}\right)=a_{0} u_{n}(x)+a_{1} v_{n}(x)+w_{n}(x)
$$

with

$$
u_{n}(x):=\sum_{k=0}^{n} A_{k}(x-c)^{k}, \quad v_{n}(x):=\sum_{k=0}^{n} B_{k}(x-c)^{k}, \quad w_{n}(x)=: \sum_{k=0}^{n} C_{k}(x-c)^{k} .
$$

For any couple $\left(a_{0}, a_{1}\right)$ we have a different problem $(2)$ with a unique solution $y\left(x ; a_{0}, a_{1}\right)$. It is also the solution of $(1)$ for a certain couple $\left(a_{0}, a_{1}\right)$, the one that satisfies the linear system $M Y=N$, with

$$
Y=\left(\begin{array}{c}
u(0) a_{0}+v(0) a_{1}+w(0) \\
u^{\prime}(0) a_{0}+v^{\prime}(0) a_{1}+w^{\prime}(0) \\
u(1) a_{0}+v(1) a_{1}+w(1) \\
u^{\prime}(1) a_{0}+v^{\prime}(1) a_{1}+w^{\prime}(1)
\end{array}\right)
$$

The approximation $\left(\tilde{a}_{0}, \tilde{a}_{1}\right)$ of the exact solution $\left(a_{0}, a_{1}\right)$ of this system constructed in Algorithm 1 is the solution of the linear system $M \tilde{Y}=N$, with

$$
\tilde{Y}=\left(\begin{array}{c}
u_{n}(0) \tilde{a}_{0}+v_{n}(0) \tilde{a}_{1}+w_{n}(0) \\
u_{n}^{\prime}(0) \tilde{a}_{0}+v_{n}^{\prime}(0) \tilde{a}_{1}+w_{n}^{\prime}(0) \\
u_{n}(1) \tilde{a}_{0}+v_{n}(1) \tilde{a}_{1}+w_{n}(1) \\
u_{n}^{\prime}(1) \tilde{a}_{0}+v_{n}^{\prime}(1) \tilde{a}_{1}+w_{n}^{\prime}(1)
\end{array}\right)
$$

Then, the approximate solution $\tilde{y}_{n}(x)$ of $(1)$ constructed in Algorithm 1 is:

$$
\tilde{y}_{n}(x)=y_{n}\left(x ; \tilde{a}_{0}, \tilde{a}_{1}\right)=\tilde{a}_{0} u_{n}(x)+\tilde{a}_{1} v_{n}(x)+w_{n}(x)
$$

which satisfies exactly the boundary conditions and approximately the differential equation. We have that $u_{n}(x) \rightarrow u(x), v_{n}(x) \rightarrow v(x)$ and $w_{n}(x) \rightarrow w(x)$ when $n \rightarrow \infty$ uniformly in $x \in[0,1]$. Then, when $n \rightarrow \infty$, the linear system $M \tilde{Y}=N$ becomes the linear system $M Y=N$, which has a unique solution $\left(a_{0}, a_{1}\right)$. Therefore, for large enough $n$, the system $M \tilde{Y}=N$ has a unique solution $\left(\tilde{a}_{0}, \tilde{a}_{1}\right)$ such that $\left(\tilde{a}_{0}, \tilde{a}_{1}\right) \rightarrow\left(a_{0}, a_{1}\right)$ when $n \rightarrow \infty$. Then, the approximate solution $\tilde{y}_{n}(x)$ constructed in Algorithm 1 approximates the unique solution $y\left(x ; a_{0}, a_{1}\right)$ of $(1)$ when $n \rightarrow \infty$ uniformly in $x \in[0,1]$ :

$$
\tilde{y}_{n}(x)=\tilde{a}_{0} u_{n}(x)+\tilde{a}_{1} v_{n}(x)+w_{n}(x) \rightarrow a_{0} u(x)+a_{1} v(x)+w(x)=y\left(x ; a_{0}, a_{1}\right)
$$

Algorithm 1 can be reformulated in the following way: 
Algorithm 2. For a given $n \in \mathbb{N}$, consider the (unknown) Taylor polynomial of $y(x)$ at $x=c$ and degree $n$ given in (6). Equating to zero the first $n-1$ Taylor coefficients of the function $R_{n}(x):=$ $\varphi(x) y_{n}^{\prime \prime}+f(x) y_{n}^{\prime}+g(x) y_{n}-h(x)$ at $x=c$ we obtain a linear system of $n-1$ equations with $n+1$ unknowns $a_{0}, a_{1}, \ldots, a_{n}$. These $n-1$ equations are just the equations (4) for $k=2,3,4, \ldots, n$. This linear system is complemented with the two linear equations $M Y=N$. The solution $\tilde{a}_{0}, \tilde{a}_{1}, \ldots, \tilde{a}_{n}$ of this complete system gives an approximation of the Taylor polynomial $y_{n}(x): y_{n}(x) \simeq \tilde{y}_{n}(x):=\sum_{k=0}^{n} \tilde{a}_{k}(x-c)^{k}$.

With Algorithm 2, we compute at once all of the coefficients $\tilde{a}_{0}, \tilde{a}_{1}, \ldots, \tilde{a}_{n}$ as the solution of a linear system of $n+1$ equations (the $n-1$ equations (4) plus the two equations $M \tilde{Y}=N$ ). With Algorithm 1, we compute first the coefficients $a_{2}, a_{3}, \ldots a_{n}$ as functions of $a_{0}$ and $a_{1}$ (the $n-1$ equations (4)). Then, we compute the approximations $\tilde{a}_{0}$ and $\tilde{a}_{1}$ from the two equations $M \tilde{Y}=N$. Finally, we obtain $\tilde{a}_{2}, \tilde{a}_{3}, \ldots, \tilde{a}_{n}$ replacing $a_{0}$ and $a_{1}$ by $\tilde{a}_{0}$ and $\tilde{a}_{1}$ respectively in (4).

The matrix method proposed in [2] and [7] to approximate $y(x)$ is a reformulation of Algorithm 2.

In the remaining of the paper, and for convenience in the notation, we replace the interval $[0,1]$ in (1) with the interval $[-1,1]$ and consider the boundary value problem:

$$
\begin{cases}\varphi(x) y^{\prime \prime}+f(x) y^{\prime}+g(x) y-h(x)=0 & \text { in }(-1,1), \\ M Y=N, & Y^{T}=\left(y(-1), y^{\prime}(-1), y(1), y^{\prime}(1)\right),\end{cases}
$$

where the rank-2 matrix $M$ and the vector $N$ are given below formula (1). We suppose that (8) has a unique solution.

\section{A Taylor expansion of the solution at the two extreme points}

In this section, instead of approximating the solution $y$ of (8) by a standard Taylor expansion, we consider a two-point Taylor expansion at the base points $x= \pm 1$ [3]. This selection of base points has some computational advantages because the boundary conditions are given at these points.

Let $\mathcal{O}_{r}$ denote the Cassini oval in the complex plane with foci at $x= \pm 1$ and Cassini radius $r$; that is, $\mathcal{O}_{r}$ is defined by $\left\{z \in \mathbb{C}|| z^{2}-1 \mid=r\right\}$. Let $\mathcal{D}_{r}$ denote the Cassini disk defined by $\left\{z \in \mathbb{C}|| z^{2}-1 \mid<r\right\}$.

When $r>1, \mathcal{O}_{r}$ is a single oval, when $r=1$ it is a lemniscate, and when $r<1$ it consists of two small ovals around the points \pm 1 . When we assume $r>1$, the interval $[-1,1]$ is lying inside $\mathcal{O}_{r}$ (see Figure 2).

Suppose that the functions $\varphi, f, g$ and $h$ are analytic in the Cassini disk $\mathcal{D}_{r}, r>1$, and $\varphi \neq 0$ in $\mathcal{D}_{r}$. We propose the following algorithm to approximate the unique solution $y$ of (8).

Algorithm 3. The method of Frobenius assures that the unique solution $y$ of (8) is analytic in the Cassini oval $\mathcal{D}_{r}$. Then, it is shown in [3], [4] that $y$ admits a two-point Taylor expansion of the form:

$$
y(x)=\sum_{k=0}^{\infty}\left[a_{k}+b_{k} x\right]\left(x^{2}-1\right)^{k},
$$

where the (unique) two-point Taylor coefficients $a_{k}$ and $b_{k}$ are related to the derivatives of $y$ at $x= \pm 1$ [3]. Apart from using the formulas given in [3], the coefficients $a_{k}$ and $b_{k}$ may be obtained recursively by replacing $y$ in the differential equation (8) by the above expansion. Also, we have to replace $y^{\prime}$ and $y^{\prime \prime}$ by

$$
\begin{aligned}
& y_{n}^{\prime}(x)=\sum_{k=0}^{\infty}\left\{\left[(2 k+1) b_{k}+2(k+1) b_{k+1}\right]+2(k+1) a_{k+1} x\right\}\left(x^{2}-1\right)^{k}, \\
& y_{n}^{\prime \prime}(x)=\sum_{k=0}^{\infty} 2(k+1)\left\{\left[(2 k+1) a_{k+1}+2(k+2) a_{k+2}\right]+\left[(2 k+3) b_{k+1}+2(k+2) b_{k+2}\right] x\right\}\left(x^{2}-1\right)^{k} .
\end{aligned}
$$


We equate to zero the two-point Taylor coefficients of $R(x):=\varphi(x) y^{\prime \prime}+f(x) y^{\prime}+g(x) y-h(x)$ at $x= \pm 1$. We obtain in this way $a_{k}$ and $b_{k}, k=2,3,4, \ldots$, from a system of two recursions of the form

$$
\left\{\begin{array}{rl}
a_{k} & =\sum_{j=0}^{k-1}\left[\alpha_{k, j} a_{j}+\beta_{k, j} b_{j}\right]+\gamma_{k}, \\
b_{k} & =\sum_{j=0}^{k-1}\left[\alpha_{k, j}^{\prime} a_{j}+\beta_{k, j}^{\prime} b_{j}\right]+\gamma_{k}^{\prime},
\end{array} \quad k=2,3,4, \ldots,\right.
$$

where the coefficients $\alpha_{k, j}, \beta_{k, j}, \gamma_{k}, \alpha_{k, j}^{\prime}, \beta_{k, j}^{\prime}, \gamma_{k}^{\prime}$ depend on the two-point Taylor coefficients of $\varphi, f$, $g$ and $h$ at $x= \pm 1$. The computation of the coefficients $a_{k}, b_{k}, k=2,3,4, \ldots$ requires the initial seed $a_{0}, b_{0}, a_{1}$ and $b_{1}$. From these recurrence relations we obtain the two-point Taylor coefficients $a_{k}$ and $b_{k}$, $k=2,3,4, \ldots$ of $y$ at $x= \pm 1$ as an affine combination of the four first coefficients $a_{0}, b_{0}, a_{1}$ and $b_{1}$ :

$$
\left\{\begin{array}{l}
a_{k}=A_{k} a_{0}+B_{k} b_{0}+C_{k} a_{1}+D_{k} b_{1}+E_{k}, \\
b_{k}=F_{k} a_{0}+G_{k} b_{0}+H_{k} a_{1}+I_{k} b_{1}+J_{k},
\end{array} \quad k=2,3,4, \ldots,\right.
$$

where the coefficients $A_{k}, B_{k}, \ldots, J_{k}$ are functions of $\alpha_{k, j}, \beta_{k, j}, \gamma_{k}, \alpha_{k, j}^{\prime}, \beta_{k, j}^{\prime}, \gamma_{k}^{\prime}$. The parameters $a_{0}, b_{0}$, $a_{1}$ and $b_{1}$ are linked by the equations $M Y=N$, with

$$
Y^{T}=\left(a_{0}-b_{0}, b_{0}+2 b_{1}-2 a_{1}, a_{0}+b_{0}, b_{0}+2 b_{1}+2 a_{1}\right)
$$

This means that only two of these four parameters are free; suppose, for example, that $a_{1}$ and $b_{1}$ are free (if we choose another couple of parameters as free parameters we can proceed in a similar manner). Then, every two-point Taylor coefficient $a_{k}$ and $b_{k}, k=2,3,4, \ldots$ is an affine combination of only $a_{1}$ and $b_{1}$.

Every couple $\left(a_{1}, b_{1}\right)$ gives rise to a different function $y$ given by (9)-(12). Formally, all of these functions $y$ are solutions of (8). But this problem has a unique solution, and then it must happen that the series (9) is convergent only for one couple $\left(a_{1}, b_{1}\right)$, the one that gives rise to the unique solution of (8). The series (9) must be divergent for any other couple $\left(a_{1}, b_{1}\right)$.

The correct values $\left(a_{1}, b_{1}\right)$ may be then obtained by imposing the convergence of (9). In practise, we obtain an approximation $\left(\tilde{a}_{1}, \tilde{b}_{1}\right)$ of $\left(a_{1}, b_{1}\right)$ by solving the two linear equations $a_{n+1}=b_{n+1}=0$ $\left(a_{n+1}\right.$ and $b_{n+1}$ are affine combinations of $a_{1}$ and $\left.b_{1}\right)$. Doing this we are imposing implicitly that (9) is convergent when we approximate this infinite series by

$$
y_{n}(x):=\sum_{k=0}^{n}\left[a_{k}+b_{k} x\right]\left(x^{2}-1\right)^{k} .
$$

Once we have obtained the approximation $\left(\tilde{a}_{1}, \tilde{b}_{1}\right)$, we obtain from $M Y=N$ an approximation $\left(\tilde{a}_{0}, \tilde{b}_{0}\right)$ of $\left(a_{0}, b_{0}\right)$ and then, from (12), we obtain the approximations $\tilde{a}_{k}$ and $\tilde{b}_{k}, k=2,3,4, \ldots$ of $a_{k}$ and $b_{k}$ as affine combinations of $\tilde{a}_{1}$ and $\tilde{b}_{1}$ and hence, the approximate two-point Taylor polynomial

$$
\tilde{y}_{n}(x):=\sum_{k=0}^{n}\left[\tilde{a}_{k}+\tilde{b}_{k} x\right]\left(x^{2}-1\right)^{k} .
$$

Example 2. Consider the boundary value problem

$$
\begin{cases}\left(x^{2}+a^{2}\right)^{2} y^{\prime \prime}+3 x\left(x^{2}+a^{2}\right) y^{\prime}+2 a^{2} y=0 & \text { in }(-1,1), \\ y(-1)=y(1)=\frac{1}{1+a^{2}}, & a>0 .\end{cases}
$$




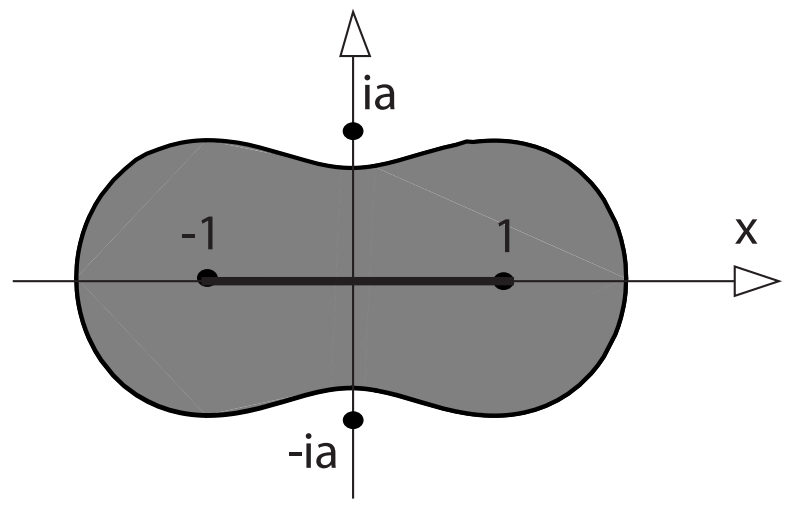

Figure 2. The Cassini disk $\mathcal{D}_{r}=\left\{z \in \mathbb{C}|| z^{2}-1 \mid<r\right\}$ with foci \pm 1 and radius $r>1$ contains the real interval $[-1,1]$. If $r<1+a^{2}$ then it does not contain the singular points $\pm i$ a of the differential equation (16).

We have $M_{11}=M_{23}=1$ and the remaining $M_{i j}=0 ; N_{1}=N_{2}=\left(1+a^{2}\right)^{-1}, \varphi(x)=\left(x^{2}+a^{2}\right)^{2}$, $f(x)=3 x\left(x^{2}+a^{2}\right), g(x)=2 a^{2}$ and $h=0$. For any $a>0$, the function $\varphi$ is nonvanishing in the Cassini disk $\mathcal{D}_{r}$ with foci at $x= \pm 1$ for any $r>1$ satisfying $r<1+a^{2}$ (see Figure 2).

We have

$$
\left\{\begin{array} { r l } 
{ y ( - 1 ) } & { = a _ { 0 } - b _ { 0 } = ( 1 + a ^ { 2 } ) ^ { - 1 } } \\
{ y ( 1 ) } & { = a _ { 0 } + b _ { 0 } = ( 1 + a ^ { 2 } ) ^ { - 1 } }
\end{array} \Longrightarrow \left\{\begin{array}{l}
a_{0}=\left(1+a^{2}\right)^{-1}, \\
b_{0}=0 .
\end{array}\right.\right.
$$

The two-point Taylor expansions of the coefficient functions are finite in this example:

$$
\begin{gathered}
\varphi(x)=\left[\left(a^{2}+1\right)^{2}+0 x\right]+\left[2\left(a^{2}+1\right)+0 x\right]\left(x^{2}-1\right)+[1+0 x]\left(x^{2}-1\right)^{2}, \\
f(x)=\left[0+3\left(a^{2}+1\right) x\right]+[0+3 x]\left(x^{2}-1\right), \quad g(x)=\left[2 a^{2}+0 x\right],
\end{gathered}
$$

and then, the recursions (11) are, for $k=0,1,2, \ldots$ and $a_{-1}=b_{-1}=0$,

$$
\begin{aligned}
& 4(k+1)(k+2)\left(a^{2}+1\right)^{2} a_{k+2}+2(k+1)\left[(2 k+1) a^{2}+2(3 k+2)\right]\left(a^{2}+1\right) a_{k+1} \\
& +2\left[\left(4 k^{2}+k+1\right) a^{2}+2 k(3 k+1)\right] a_{k}+4 k(k-1) a_{k-1}=0, \\
& 4(k+1)(k+2)\left(a^{2}+1\right)^{2} b_{k+2}+2(k+1)\left[(2 k+3) a^{2}+6(k+1)\right]\left(a^{2}+1\right) b_{k+1} \\
& +\left[((4 k+3)(2 k+1)+2) a^{2}+3(2 k+1)^{2}\right] b_{k}+\left(4 k^{2}-1\right) b_{k-1}=0,
\end{aligned}
$$

with $a_{0}$ and $b_{0}$ given above and $a_{1}$ and $b_{1}$ free.

For several values of $n \in \mathbb{N}$, we solve the equations $a_{n+1}=b_{n+1}=0$ for $a_{1}$ and $b_{1}$ and obtain the approximate values $\tilde{a}_{1}$ and $\tilde{b}_{1}$. From the above recursions and using the obtained (exact) values of $a_{0}$ and $b_{0}$ and the approximate $\tilde{a}_{1}$ and $\tilde{b}_{1}$ we obtain the approximate Taylor polynomial (15). Figure 3 shows the approximation $\tilde{y}_{n}(x)$ of $y(x)$ for some values of $n$ and $a$.

Observe that if $a \leq 1$ we cannot apply the method of Section 2 because the function $\varphi(x)$ vanishes at $x= \pm i a$, which points are inside any circle containing the interval $[-1,1]$. In fact, the exact (and unique) solution of (7) is the function $y(x)=\left(x^{2}+a^{2}\right)^{-1}$ which is singular at $x= \pm i a$. 


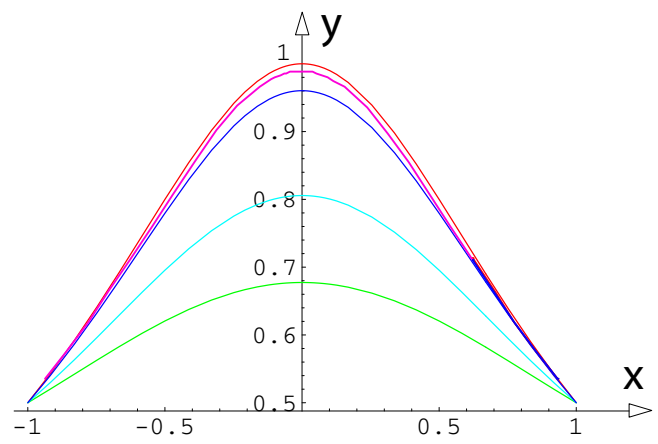

Figure 3. Plot of the exact solution $y(x)=\left(x^{2}+a^{2}\right)^{-1}$ (red) of (16) and the approximations $\tilde{y}_{3}(x)$ (green), $\tilde{y}_{5}(x)$ (blue), $\tilde{y}_{9}(x)$ (dark blue) and $\tilde{y}_{11}(x)$ (purple) with $a=1$.

Algorithm 3 can be reformulated in the following way:

Algorithm 4. For a given $n \in \mathbb{N}$, consider the two-point Taylor polynomial of degree $n, y_{n}$ given in (14) and its derivatives $y_{n}^{\prime}$ and $y_{n}^{\prime \prime}$ given by the series (10) truncated at $k=n$. Equating to zero the first $2 n$ two-point Taylor coefficients of the function $R_{n}(x):=\varphi(x) y_{n}^{\prime \prime}+f(x) y_{n}^{\prime}+g(x) y_{n}-h(x)$ we obtain $2 n$ linear equations for the $2(n+1)$ unknowns $a_{0}, a_{1}, \ldots, a_{n} ; b_{0}, b_{1}, \ldots, b_{n}$. These $2 n$ linear equations are supplemented with the two linear equations $M Y=N$, with $Y$ given in (13). The solution of this linear system of $2(n+1)$ equations with $2(n+1)$ unknowns gives an approximation $\tilde{a}_{k}, \tilde{b}_{k}, k=0,1,2, \ldots, n$, of $a_{k}, b_{k}$, and hence an approximation $\tilde{y}_{n}(x)$ of the two-point Taylor polynomial $y_{n}(x)$ of the solution $y(x)$ of (8).

Observe that $R_{n}(x)=\mathcal{O}\left((x \pm 1)^{n-1}\right)$ as $x \rightarrow \pm 1$. From this fact we deduce immediately a third algorithm to approximate $y_{n}$ more appropriate for a computer algebraic manipulator:

Algorithm 5. The $n$ first coefficients of the standard Taylor expansion of $R_{n}$ at $x=1$ and also at $x=-1$ vanish. Then, equate to zero the first $n$ Taylor coefficients of $R_{n}$ at $x=-1$ and the first $n$ Taylor coefficients of $R_{n}(x)$ at $x=1$. Consider also the two linear equations $M Y=N$. From these $2 n+2$ linear equations we can obtain an approximation $\tilde{a}_{k}, \tilde{b}_{k}, k=0,1,2, \ldots, n$, of the first $2 n+2$ two-point Taylor coefficients $a_{k}$ and $b_{k}$ of $y_{n}(x)$.

\section{A Taylor expansion of the solution at $n$ points}

When the Cassini disk $\mathcal{D}_{r}$ of analyticity of the coefficient functions of (8) with foci $x= \pm 1$ does not contain the interval $[-1,1]$, we may consider a $n$-point Taylor expansion with $n>2$ [4]. When those base points are conveniently chosen, we facilitate the inclusion of the interval $[-1,1]$ in the generalized Cassini disk of convergence of the $n$-point Taylor expansion. The generalization of the three algorithms explained in the above section is straightforward. We just illustrate the idea for $n=3$ with the following example.

Example 3. Consider the boundary value problem

$$
\left\{\begin{array}{l}
{\left[(x+1 / 2)^{2}+a^{2}\right]\left[(x-1 / 2)^{2}+a^{2}\right] y^{\prime \prime}} \\
+4\left\{(x+1 / 2)\left[(x-1 / 2)^{2}+a^{2}\right]+(x-1 / 2)\left[(x+1 / 2)^{2}+a^{2}\right]\right\} y^{\prime} \\
+2\left\{\left[(x+1 / 2)^{2}+a^{2}\right]+\left[(x-1 / 2)^{2}+a^{2}\right]+4\left(x^{2}-1 / 4\right)\right\} y=0 \quad \text { in }(-1,1), \\
y(-1)=y(1)=\frac{1}{\left(1 / 4+a^{2}\right)\left(9 / 4+a^{2}\right)}, \quad a>0 .
\end{array}\right.
$$


The coefficient functions are entire functions, but the function $\varphi(x)=\left[(x+1 / 2)^{2}+a^{2}\right]\left[(x-1 / 2)^{2}+a^{2}\right]$ vanishes at $x= \pm 1 / 2 \pm i a$. If $a<\sqrt{\sqrt{2}-5 / 4}$, we cannot find a Cassini oval with foci at $x= \pm 1$ that contains the interval $[-1,1]$ and that does not contain the points $x= \pm 1 / 2 \pm i a[3]$. Hence, we cannot apply the method of Section 3. Consider then a 3-point Taylor approximation for $y$ with base points $x= \pm 1$ and $x=0[4]$ :

$$
y(x)=\sum_{k=0}^{\infty}\left[a_{k}+b_{k} x+c_{k} x^{2}\right] x^{k}\left(x^{2}-1\right)^{k} .
$$

This expansion is convergent in the region [4]:

$$
\mathcal{E}_{r}=\left\{z \in \mathbb{C}|| z\left(z^{2}-1\right) \mid<r\right\}, \quad r \leq\left(\frac{1}{4}+a^{2}\right) \sqrt{\frac{9}{4}+a^{2}},
$$

that does not contain the points $x= \pm 1 / 2 \pm i a$. Moreover, this region contains the interval $[-1,1]$ when $r>2 /(3 \sqrt{3})$ (see Figure 4).

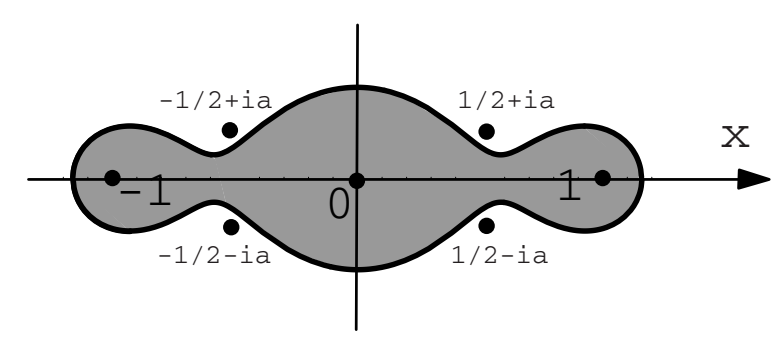

Figure 4. The region $\mathcal{E}_{r}$ of convergence given in of (19) of the expansion (18) contains the real interval $[-1,1]$ if $r>2 /(3 \sqrt{3})$. If $r \leq\left(1 / 4+a^{2}\right) \sqrt{9 / 4+a^{2}}$ then it does not contain the zeros $\pm 1 / 2 \pm i$ a of the function $\varphi$ of Example 3.

A straightforward generalization of Algorithm 4 to the case with three points is the following.

Algorithm 4'. For several values of $n \in \mathbb{N}$, we replace $y$ in the differential equation (17) by its three-point Taylor polynomial

$$
y_{n}(x):=\sum_{k=0}^{n}\left[a_{k}+b_{k} x+c_{k} x^{2}\right] x^{k}\left(x^{2}-1\right)^{k} .
$$

Then, the approximate $3 n$ coefficients $\tilde{a}_{0}, \tilde{b}_{0}, \tilde{c}_{0} \ldots, \tilde{a}_{n}, \tilde{b}_{n}, \tilde{c}_{n}$ follow from equating to zero the first $3 n+1$ three-point Taylor coefficients of the function $R_{n}(x):=\varphi(x) y_{n}^{\prime \prime}+f(x) y_{n}^{\prime}+g(x) y_{n}-h(x)$ and from the boundary conditions $M Y_{n}=N$, with

$$
Y^{T}=\left(a_{0}-b_{0}+c_{0}, b_{0}-2 c_{0}+2 a_{1}-2 b_{1}+2 c_{1}, a_{0}+b_{0}+c_{0}, b_{0}+2 c_{0}+2 a_{1}+2 b_{1}+2 c_{1}\right) .
$$

Figure 5 shows the approximation for some values of $n$ and $a$.

A straightforward generalization of Algorithm 5 to the case of three points is the following.

Algorithm 5'. Equate to zero the first $n$ Taylor coefficients of $R_{n}$ at $x=-1$, its first $n+1$ Taylor coefficients at $x=0$ and its the first $n$ Taylor coefficients of at $x=1$. Consider also the two linear equations $M Y=N$. From these $3 n+3$ linear equations we obtain an approximation $\tilde{a}_{k}, \tilde{b}_{k}$ and $\tilde{c}_{k}$, $k=0,1,2, \ldots, n$ to the first $3 n+3$ three-point Taylor coefficients $a_{k}, b_{k}$ and $c_{k}, k=0,1,2, \ldots, n$ of $y_{n}(x)$. 


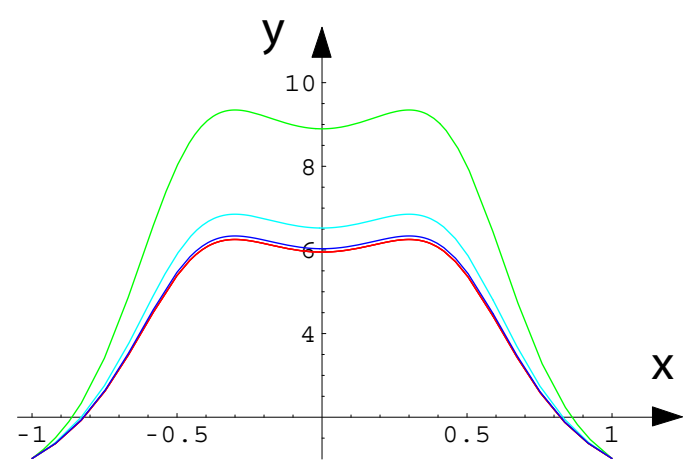

Figure 5. Plot of the exact solution $y(x)=\left\{\left[(x+1 / 2)^{2}+a^{2}\right]\left[(x-1 / 2)^{2}+a^{2}\right]\right\}^{-1}$ (red) of (17) and the approximations $\tilde{y}_{5}(x)$ (green), $\tilde{y}_{10}(x)$ (blue), $\tilde{y}_{15}(x)$ (dark blue) with $a=0.4$.

If the coefficient functions have more singular points $P_{1}, P_{2}, P_{3}, \ldots$ close to the interval $[-1,1]$ then we should consider a multi-point Taylor expansion with more base points such that the region of convergence avoids those singular points and contains the interval $[-1,1]$. When we take more base points for the multipoint Taylor expansion, we squeeze the convergence region of the expansion avoiding the singular points $P_{k}$ and facilitate the inclusion of the interval $[-1,1]$ in this region [4] (see Figure 6). The generalization of the above algorithms to the $n$-points Taylor expansion case is straightforward.

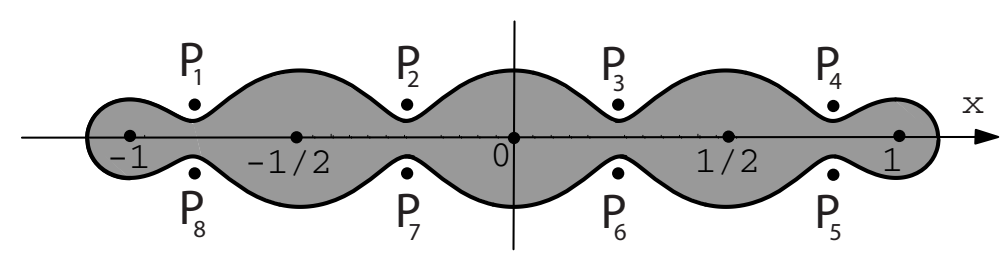

Figure 6. Typical portrait of the convergence region of a 5-point Taylor expansion at the five base points $x= \pm 1$, $x= \pm 1 / 2$ and $x=0$.

\section{Concluding remarks}

When one of the function coefficients of the differential equation in (1) is not analytic and/or $\varphi$ is vanishing inside any circle containing the interval $[0,1]$, we cannot use the standard Taylor expansion [2], [7] to approximate the solution of (1). In this case we can subdivide the interval $[0,1]$ and approximate the solution in every subinterval by Taylor expansions that must be matched on overlapping intervals [[5], Sec. 7]. We obtain in this way an approximation of the solution of (1) in the form of a piecewise polynomial in several subintervals of $[0,1]$.

In this paper we have proposed an alternative to the method considered in [2] that simplifies the computations. Moreover, we have shown its convergence. Algorithm 2 proposed in Section 2 is a different version of the Taylor-matrix method proposed in [2], [7] and has some resemblance with the method proposed in [1] to approximate the solution of Fredholm integral equations.

The method introduced in [5] to approximate the solution of (8) gives an analytic approximation to the solution, but this approximation is not uniform in the whole interval $[-1,1]$ and the successive matching of expansions translates into numerical errors. In Section 3 we have proposed an alternative 
to this method by considering the Taylor expansion of the solution $y$ simultaneously at $x= \pm 1$. With this procedure we obtain a convergent expansion of the solution uniformly valid in the interval $[-1,1]$. When the Cassini disk of convergence of the coefficients of the differential equation does not contain the interval $[-1,1]$ we may consider the Taylor expansion of the solution, not only at $x= \pm 1$, but also at other intermediate points (Section 4). With this multi-point Taylor expansion we squeeze the convergence region of the expansion in order to avoid singular points and to include the whole interval $[-1,1]$.

In contrast with the standard method of Frobenius for initial value problems, the coefficients of the polynomial approximations obtained with these methods are not the exact Taylor coefficients of the approximated functions, but approximations of those coefficients that get closer and closer to the exact coefficients when the degree $n$ of the approximation increases.

\section{Acknowledgments}

JLL and EPS acknowledge financial support from Dirección General de Ciencia y Tecnología, project MTM2004-05221. NMT acknowledges financial support from Ministerio de Educación y Ciencia, project MTM2006-09050. 


\section{References}

[1] R.P. Kanwall and K.C. LiU, A Taylor expansion approach for solving integral equations, Int. J. Math. Edu. Sci. Technol., 20 (1989), 411-414.

[2] C. Kesan, Taylor polynomial solutions of linear differential equations, Appl. Math. Comp., 142 (2003), no. 1, 155-165.

[3] J.L. López and Nico M. Temme, Two-point Taylor expansions of analytic functions, Stud. Appl. Math., 109 (2002), no. 4, 297-311.

[4] J.L. López And Nico M. Temme, Multi-point Taylor expansions of analytic functions, Trans. Amer. Math. Soc., 356 (2004), no. 11, 4323-4342 (electronic).

[5] A. B. Olde DaAlhuis and F. W. J. Olver, On the asymptotic and numerical solution of linear ordinary differential equations, SIAM Rev., 40 (1998), no. 3, 463-495.

[6] A. D. Polyanin and V. F. Zaitsev, Handbook of Exact Solutions for Ordinary Differential equations, Chapman \& Hall/CRC, New York, 2002.

[7] M. SEzER, A method for the approximate solution of the second-order linear differential equations in terms of Taylor polynomials, Int. J. Math. Edu. Sci. Technol., 27 (1996), 821-834.

[8] N.M. Temme, Special functions: An introduction to the classical functions of mathematical physics, Wiley and Sons, New York, 1996. 